\title{
La variable ideológica como elemento central para la constitución y desarrollo de capital social
}

\author{
Jorge Brower Beltramin
}

Universidad de Santiago de Chile, Santiago, Chile. Email: jorge.brower@usach.cl

Resumen: el concepto de capital social ha sido abordado con gran interés en las últimas décadas por la sociología y las teorías del desarrollo en general. Todas las investigaciones tanto teóricas como aplicadas, reconocen en esta forma de capital la ayuda recíproca y la cooperación como sus componentes fundamentales. Este artículo tiene por objetivo, desarrollar una reflexión respecto a la importancia de la variable ideológica para la constitución y desarrollo de capital social. En este contexto, la literatura especializada concuerda en la necesidad de que existan valores, creencias y normas a partir de las cuales se articulen las redes de relaciones recíprocas y cooperativas. Estos elementos constituyen ideologías que, desde nuestra perspectiva, aseguran la formación de capital social que refuerza desde las bases de la sociedad, el funcionamiento pleno de la democracia.

Palabras clave: capital social, ideología, reciprocidad, confianza.

\section{The ideological variable as central element for the constitution and development of social capital}

\begin{abstract}
: the concept of social capital has been boarded with great interest in the last decades by sociology and in general by the theories on development. The theoretical and applied investigations recognize in this form of capital the reciprocal aid and cooperation as their fundamental components. This article has by objective, to develop an analysis in regards to the importance of the ideological variable for the constitution and development of social capital. Against this background, the specialized literature agrees in the necessity that values, beliefs and norms exist, from which the networks of reciprocal and cooperative relations articulate. These elements constitute ideologies that, from our perspective, assure the formation of social capital that reinforces from the bases of the society, the the complete functioning of democracy.
\end{abstract}

Key words: social capital, ideology, reciprocity, confidence.

\section{A variável ideológica como central para a criação e desenvolvimento de capital social}

Resumo: O conceito de capital social tem sido abordado com grande interesse nas últimas décadas em sociologia e as teorias do desenvolvimento em geral. Todas as pesquisas teóricas e aplicadas, reconhecer capital nesta forma de assistência mútua e cooperação como seus componentes fundamentais. Este artigo tem como objetivo desenvolver uma reflexão sobre a importância da variável ideo- 
lógica para a criação e desenvolvimento de capital social. Neste contexto, a literatura concorda com a necessidade de valores, crenças e normas a partir do qual a articular as redes de relações recíprocas e cooperativo. Estes elementos são ideologias que, a partir de nossa perspectiva, garante a formação de capital social que fortalece a partir da base da sociedade, o pleno funcionamento da democracia.

Palavras-chave: capital social, a ideologia, a reciprocidade, confiança.

\section{Aproximación al concepto de capital social}

La conceptualización teórica sobre el capital social ha tenido un vertiginoso desarrollo desde mediados de la década de los 80’ del siglo pasado. Este trabajo, esencialmente académico, vinculado a procesos sociales emergentes y otros de más larga data, ha generado un volumen discursivo de gran densidad, esforzándose por definir este fenómeno y comprenderlo, desde perspectivas disciplinares distintas, en ámbitos socioculturales también de muy diversas características.

En este artículo, pretendemos detenernos en una de las variables a considerar, como elemento fundamental para el desarrollo de capital social, esta es la ideología, en un sentido amplio que explicaremos luego. Dicha variable, es considerada por los teóricos que han abordado esta problemática particular, pero creemos que es posible avanzar respecto de su importancia para el incremento de esta forma de capital en un contexto globalizado que tiende a debilitar los contenidos fundamentales generados al interior de una cultura, en los que se encuentran sus valores y creencias esenciales, ligados no sólo a los individuos que forman parte de una comunidad, sino que a los escenarios naturales en que esas comunidades viven.

Uno de los investigadores que instaló con fuerza el tema del capital social en los años 90’, dentro de la agenda de la sociología del desarrollo, es R. Putnam. Para este autor se trataría de ciertas características de las organizaciones sociales referidas a estructuras de relaciones interpersonales y también intersectoriales, expresadas a través de normas y vínculos de confianza entre los implicados en esas relaciones (Putnam 1993). En esta perspectiva uno de los elementos esenciales a considerar en términos iniciales, tiene que ver con la existencia de ciertas normas de reciprocidad amplias constituidas por obligaciones, valores y expectativas de quienes participan en diferentes tipos de grupos o asociaciones cívicas. A partir de estas consideraciones se establecerían relaciones de intercambio basadas en acuerdos previos que producen resultados concretos a corto mediano y largo plazo. (Putnam 1993). ${ }^{1}$

Desde el punto de vista de este autor, son las normas compartidas ya aceptadas por los miembros de la sociedad y de las comunidades particulares, las que instalan las reglas del juego a partir de las cuales se lleva a cabo la cooperación, estableciendo simultáneamente un sentido de lo cívico que 
se expresa en acciones conjuntas cuyo horizonte es alcanzar objetivos comunes. Así, sólo esta instalación normativa compartida es la que permite el real desarrollo y crecimiento del capital social, que se manifiesta en un conjunto de redes de compromiso cívico, cuya complejidad tiene que ver con los ámbitos sociales involucrados, los actores que forman parte de dichos ámbitos y el tipo de contenidos que circula en estas redes interconectadas a partir de un conjunto de reglas del juego conocidas y respetadas, como ya hemos señalado.

Dentro de la concepción de Putnam sobre el capital social, el funcionamiento de las redes de compromiso y el logro efectivo de ciertos objetivos trazados por los grupos comunitarios, agrega a este capital social, la confianza, entendida como valor que se desprende de la correcta aplicación de las normas de reciprocidad a las que ya henos hecho referencia. De este modo se completa un verdadero circuito que da cuenta de un capital social cuya densidad tiene que ver con la calidad de las redes de reciprocidad establecidas, con la historia y experiencia de la puesta a prueba de esas redes, en definitiva, con el crecimiento de las comunidades orientadas por objetivos de desarrollo compartidos.

En términos globales, Putnam concibe el capital social como la capacidad efectiva de concretar iniciativas locales en todos los ámbitos (político, económico, deportivo, etc.) incluyendo por cierto, la resolución de necesidades y problemas, mediante estas redes interpersonales. Por último, los distintos niveles de capital social articulan sociedades cívicas que fortalecen y validan los gobiernos democráticos, sobre una plataforma de sociedad civil activa y protagonista de su propio desarrollo.

La conceptualización formal sobre el capital social desarrollada por Putnam, aborda a nuestro juicio, los elementos esenciales que deben ser considerados para una revisión comprensiva de este fenómeno. Más allá de consideraciones teóricas provenientes de diferentes estructuras epistémicas, otros autores hacen su aporte, sin ser estos excluyentes respecto de los planteamientos ya realizados en este trabajo. Es el caso, en primer término, de Bourdieu, quien ya a comienzo de los 80' se refería al capital social no sólo como el conjunto de propiedades comunes dentro de un grupo y su capacidad de articularse con otros, sino que ponía el énfasis en que dichas redes debían proyectarse como permanentes y útiles más allá de una comprensión objetiva de las mismas. De igual forma, este investigador atribuye gran importancia a la articulación material y simbólica de este tipo de vinculación, siendo estos elementos, claves esenciales para visualizar la real proximidad de los grupos que interactúan en el espacio social. (Bourdieu 1980) Cabe destacar también, en la perspectiva de esta autor, la importancia asignada a cada sujeto, entendido como guardián de los límites de su grupo. Esta afirmación implica un conocimiento prolongado y maduro de las normas y valores que regulan las relaciones interpersonales, así como de los otros miembros del grupo, conocimiento que da estabilidad a las relaciones y aumenta el capital social en torno al valor de la confianza, como ya exponíamos a propósito de los planteamientos de Putnam. ${ }^{2}$ 
Volviendo a los aportes de Bourdieu (1985), sobre las características del capital social, insiste en el establecimiento de una red durable de relaciones en que se produce un reconocimiento de los individuos que interaccionan en el ámbito del desarrollo social. Esta afirmación ratifica lo antes expuesto y se vincula directamente con el incremento del valor de la confianza como producto de la experiencia en el tiempo y los resultados de ésta en términos de los fines establecidos por una comunidad. Esta postura es enriquecida también por Coleman (1990), otro sociólogo que concibe el capital social como una forma de activo que posibilita de mejor manera, la interacción de los individuos al interior de una estructura social. Debemos señalar que este tipo de activo reenvía inevitablemente a los elementos o variables que ya hemos mencionado a propósito de los planteamientos de Putnam y que tiene que ver en primer término, con la existencia de normas y valores desde los cuales se establecen las relaciones de reciprocidad necesarias para el desarrollo comunitario o social.

Sin querer abundar demasiado en la caracterización conceptual respecto al capital social, con el fin de ir delimitando su articulación teórica y la importancia que la variable ideológica juega en dicha articulación, enunciaremos de manera sintética dos aportes adicionales que nos parecen significativos para este trabajo.

El primero de ellos tiene que ver con la postura de North (1990), desde el ámbito disciplinar de la economía, en relación al capital social. Para este autor, su reflexión parte de la comprensión de las instituciones actuales, como un conjunto de normas y valores que posibilitan la confianza entre los sujetos que forman parte de un grupo o de una red de relaciones en diferentes ámbitos. Para North, estos elementos (normas, valores y confianza), se expresan de manera concreta en las organizaciones como una manifestación visible de cooperación y confianza. Como es posible observar, este autor vuelve sobre normas y valores como elementos esenciales en la generación del capital social.

El segundo aporte que completa esta visión panorámica sobre capital social tiene que ver con la perspectiva de Granovetter (1985) desde la sociología económica. Para este investigador, las interacciones económicas llevadas a cabo por sujetos concretos están vinculadas e insertas en relaciones más amplias que a su vez forman parte de estructuras sociales complejas por su propia composición. A esto agrega que el comportamiento de las personas en torno a objetivos económicos, también tiene relación con elementos propios de la sociabilidad, el status y la obtención o pérdida del poder.

Ambas perspectivas (la de North y Granovetter), entregan un marco contextual a la expresión del capital social y su desarrollo. Queda claro, en conjunto con los otros aportes expuestos, que la creación y funcionamiento de redes de relación cooperativas en cualquier ámbito social, se vincula de manera compleja con múltiples variables culturales que las atraviesan y las hacen más o menos exitosas. 


\section{Importancia de la variable ideológica para la constitución y desarrollo de capital social}

A partir de la revisión sintética de aportes relevantes sobre la comprensión de lo que denominamos capital social, es posible afirmar que éste es determinado inicial y sustantivamente por un conjunto de valores y normas internalizadas en los miembros de un grupo que interactúan entre sí por períodos más bien largos, dando lugar a la generación de reglas y leyes conocidas y compartidas por ese grupo.

A lo anterior, podemos agregar que estas redes de interacciones se producen en la cultura y a través de elementos propios de ella. Sólo desde ciertas condiciones de producción de las relaciones de reciprocidad para el desarrollo social, es posible que éste se produzca, y esas condiciones están dadas por competencias culturales particulares que posibilitan una mayor o menor densidad del capital social en comunidades o sociedades humanas. De lo anterior se desprende que el valor de la confianza, sobre el cual ya hemos expuesto, es producto, inicialmente, de la existencia de contenidos éticos y morales que obligan a las partes que interactúan a respetar las normas y leyes establecidas. ${ }^{3}$

De este modo, la cultura comprendida como una articulación compleja de contenidos que regula la vida de los hombres en comunidades y sociedades más amplias, se constituye necesariamente como el referente más o menos explícito desde el cual se construyen las relaciones sociales en general, entregando valores, creencias y normas, pero también las tecnologías que posibilitan la materialización de muchos de los objetivos fijados por el grupo. De allí, la importancia de la existencia de estos insumos culturales, dentro de los cuales los contendidos éticos y morales juegan un papel central. El capital social aumenta, se hace más denso, en la medida en que se genera una certidumbre ética y moral que es previa a las interacciones y movimientos de intercambio dentro de los grupos humanos. Se instala de esta forma el valor de la confianza, finalmente porque un conjunto de expectativas se han cumplido de igual forma que en experiencias del pasado. En esta dirección concordamos con Barber (1983), quien señala que la confianza tiene como uno de sus fundamentos, el cumplimiento de la expectativa relacionada con la implementación de un orden social deseado, posibilitando con ello una mejor proyección del futuro. ${ }^{4}$

El establecimiento de un orden social anhelado por un grupo humano, significa, desde nuestra perspectiva, la validación de un conjunto de valores y creencias desarrolladas por el propio grupo. Ese conjunto de valores y creencias son el núcleo de una ideología. En tal sentido, creemos al igual que Coleman (1994), que la ideología entendida como una visión de mundo, expresada de manara clara y compartida, representa una variable fundamental para la acción colectiva y en definitiva, el desarrollo de capital social. A la postre, la confianza sería el producto de un proceso de intercambios exitosos, asumidos desde una plataforma ideológica que provee 
de los valores, creencias y normas fundamentales que regulan y determinan el funcionamiento de esas redes sociales.

Así, el valor de la confianza, tan destacado por todos los autores que han teorizado en torno al capital social, se vincula directamente, desde nuestra perspectiva, al hecho de conocer y compartir una visión de mundo (weltanschauung), cuyos contenidos den cuenta de una sociedad deseada, y con un orden establecido que disminuya la incertidumbre en relación al desarrollo presente y futuro de esa sociedad. El compartir creencias y una visión de mundo contenidas en una ideología son la base para la constitución básica de capital social y su desarrollo, tendiente a una densidad que asegure que las operaciones de intercambio entre redes de individuos, sea cada vez más exitosa.

La centralidad o importancia esencial de la existencia de una plataforma ideológica definida y compartida, es destacada por teóricos recientes. Es el caso de Adler y Kwon (1999), para quienes, los valores, su expresión en sistemas simbólicos reconocibles e interpretables, en el contexto más amplio de visiones de mundo e interpretaciones que son compartidas, resultan esenciales para una buena comunicación entre las comunidades y grupos, y en nuestra opinión, son el basamento para la puesta en marcha de un capital social que se traduce en experiencia de reciprocidad y finalmente se establece como valor perdurable en el tiempo.

Nuestra postura, respecto a la importancia de que exista una variable ideológica bien definida y compartida, para poner en acción las redes de intercambio en vías de un desarrollo comunitario y también en otros niveles, ha sido reconocida y ubicada dentro de lo que Uphoff y Wijayaratna (2000) denominan capital social cognitivo. Con ello se refieren a que las creencias, valores y actitudes representan el activo de un capital social cognitivo que se articula y constituye a partir de ideales respecto del tipo de sociedad deseada, como elemento previo y esencial a las acciones más tangibles y que ya entran en el terreno propio del intercambio real.

Avanzando un poco más en la importancia de la variable ideológica para la constitución de capital social, podemos señalar que la forma en que se percibe el mundo, propia de una ideología específica, incluye necesariamente los intereses de ese grupo o sociedad y por tanto el eje ideológico se convierte en un elemento esencial, que a juicio de Coleman (1994), regula la posibilidad de creación o destrucción de capital social. En este contexto, es precisamente la ideología, entendida como una visión de mundo que contiene normas, valores y creencias específicas, la que posibilita que los individuos en circunstancia comunitarias, actúen más allá de intereses personales, orientando sus esfuerzos hacia las necesidades de un colectivo.

De este modo, el componente ideológico, al proponer una construcción de realidad y de organización de dicha realidad, orienta o dirige el accionar del grupo, funcionando como dispositivo conceptual que establece el tipo de relaciones interpersonales, y cómo deben actuar las redes de 
intercambio, promoviendo de esta forma, modalidades de relaciones o evoluciones en los tipos de vinculación que funcionan en este ámbito de la reciprocidad.

En términos globales, la variable ideológica determina cuándo las acciones colectivas son mutuamente beneficiosas, cuándo son buenas o malas y en definitiva, si corresponden a estados progresivos de desarrollo. En esta determinación o sanción regida por el dispositivo ideológico, las distinciones morales y éticas, a las cuales hacíamos referencia antes en este artículo, también son parte de los contenidos que aporta la ideología y en tal sentido la calificación de los tipos de desarrollo, como buenos o malos, constructivos o destructivos, dependen finalmente del referente ideológico desde el cual se construyen esas formas de desarrollo.

Establecida la relevancia del componente ideológico para la constitución y desarrollo de capital social, nos parece interesante adentrarnos en ciertas distinciones teóricas que ayudan a mejor comprender la función de la ideología en el establecimiento de redes de relación recíproca cooperativas. A propósito de las investigaciones de Scott (1985) respecto a las ideologías campesinas y su estructura de base, cuyos contenidos vinculados al poder y la explotación se expresan, a través de la denominación - weapons of the weak- (armas de los débiles), más los estudios de Rudé (1980) relacionadas con las ideologías de los pobres y desposeídos, concordamos en que estos sectores sociales generan estructuras ideológicas básicas, producto de la experiencia y por tanto, de los distintos tipos de interacción que han tenido por períodos muy prolongados. Estas estructuras ideológicas básicas corresponderían a lo que Rudé (1980) denomina ideologías inherentes, articuladas por grupos específicos cuyo fin es la movilización social y la defensa de sus derechos. Sin embargo, en muchas ocasiones, estas ideologías inherentes no contienen los elementos de negociación necesarios para llevar a cabo exitosas redes de intercambio beneficiosas para los intereses de grupos determinados. Por tal motivo, en la perspectiva de Rudé (1980), las ideologías inherentes de los pobres y oprimidos se pueden ver potenciadas por sistemas ideológicos de mayor densidad conceptual y sofisticación para el establecimiento de las reglas del juego en la praxis del intercambio cooperativo. Este enriquecimiento corresponde a lo que Rudé (1980) denomina como aporte de las ideologías derivadas. La función de estas ideologías es de ayudar a articular mejor el ideario básico de los grupos sociales más pobres y desposeídos, contribuyendo en la articulación efectiva de sistemas simples de pensamiento que en definitiva deben traducirse en protocolos de intercambio cooperativo en el horizonte más amplio de la defensa de sus derechos dentro de un sistema social explotador, hegemónico y dominante.

Los casos estudiados por Scott y Rudé y su teorización, no hacen más que profundizar nuestra postura respecto de la relevancia de la variable ideológica para el desarrollo de capital social. La ideología no sólo es un contenedor de valores, creencias y normas para el establecimiento de las reglas del juego cooperativo y de intercambio, sino que además incluye los 
programas de acción, respecto a cómo esos valores y creencias deben entrar en la dinámica social para una interacción que finalice en la consecución de los objetivos planteados por un grupo social. ${ }^{5}$

Desde otra perspectiva, los aportes teóricos y aplicados de van Dijk (2008), en el ámbito de la lingüística de los discursos y su vinculación con una estructura ideológica determinada, entregan una producción de conocimiento que enriquece la argumentación respecto de lo fundamental que resulta ser la variable ideológica en el desarrollo de capital social. Para este teórico del lenguaje, las ideologías, por una parte, se explicitan en las relaciones sociales a través de las interacciones comunicativas generadas por los actores-miembros de una comunidad, mediante la materialidad de los discursos y sus correspondientes estructuras semánticas. Estas estructuras semánticas son fundamentales ya que explican las funciones ideológicas en términos sociocognitivos y sus posteriores consecuencias en la praxis interactiva de las redes sociales. La perspectiva de van Dijk sobre las ideologías, nos parece relevante para los objetivos de este artículo (argumentación al menos inicial de la relevancia de la ideología para la constitución de capital social), ya que en primer término, afirma que éstas son cognitivas. Sin soslayar su condición social y su relación con estructuras sociales, sostiene que estas condiciones se producen desde objetos mentales como ideas, creencias y valores que constituyen sistemas de creencias. En tal dirección, las ideologías pueden ser consideradas como las bases axiomáticas de los sistemas de creencias que se comparten en los grupos humanos, y desde los cuales se establecen las relaciones de cooperación y reciprocidad que van dando forma al activo del capital social. Así, las ideologías se comparten como representaciones sociales (Aebischer et al., 1991), intercambiando sistemas de significación parciales que son funcionales a los objetivos trazados por un grupo que se relaciona en términos cooperativos.

En segundo lugar, y creemos que esta consideración es muy relevante en el contexto de nuestro artículo, van Dijk plantea que las ideologías son sociocognitivas, como anunciábamos antes. Esto significa que los sistemas de creencias funcionan como una interfaz entre lo cognitivo y lo social. Dicha afirmación se traduce en el hecho de que las ideologías se comparten por los miembros de un grupo social, haciendo de esta acción un elemento fundamental, ya que en esa instancia los individuos se familiarizan con los contenidos más relevantes de una visión de mundo particular, pero simultáneamente van enriqueciendo esos contenidos y modificándolos de forma tal que sean los más apropiados para dar cuenta del orden social deseado. Nuevamente, el capital social que se va constituyendo, se fortalece y densifica con esta actividad, que es permanente en el desarrollo de las sociedades humanas. Entonces, el carácter social de las ideologías se vuelve fundamental ya que su dinamización llevada a cabo por los miembros de los grupos se encuentra íntimamente ligada con los diversos tipos de intereses de dichos grupos. En esta instancia de circulación de las ideologías en las redes sociales se encuentra regulada por marcos interpretativos que permiten la intelegibilización de la realidad social cons- 
truida, incluyendo ciertamente como señala Button (1991), las relaciones con otros grupos, tan importantes en nuestros días, a propósito de la coexistencia y diálogo intercultural. Estos marcos interpretativos son claves, ya que su conocimiento estructural y contextual permite una mayor fluidez en las relaciones de cooperación para el desarrollo en cualquiera de sus niveles.

A partir de las cualidades cognitivas y sociales de los sistemas de creencias ideológicos propuestas por van Dijk, se desprenden otras características de las visiones de mundo, que nos interesa indicar sintéticamente a propósito de su importancia para el desarrollo de capital social. Para comenzar, estas bases axiomáticas, como señala van Dijk (2008) no poseen un estatus ontológico o esencialista en sí mismas, es decir, no pueden ser definidas como verdaderas o falsas, sino que han sido generadas como un explicandum vinculado directamente a condiciones de vida y proyectos de ordenamiento social, en un diálogo que en nuestros días, resulta inevitable, con otros proyectos de articulación cultural y social. De este modo, la verdad o falsedad de una ideología no constituye una problemática teórica ni práctica, haciendo que lo relevante de ésta tenga que ver con su grado de eficiencia en las instancias de conseguir objetivos planteados en una comunidad. Ese grado de eficiencia se manifiesta de manera explícita en el funcionamiento de las redes sociales que trabajan para conseguir metas puntuales en contextos sociales también específicos.

Otra consecuencia del planteamiento hecho por van Dijk sobre las ideologías, tiene que ver con su grado de complejidad. Estos grados de complejidad se relacionan con la estratificación social y las reglas sociales que se expresan de manera muy nítida en las elites o los intelectuales. En esos niveles sociales es donde se encuentran los ideólogos, quienes hacen que los sistemas ideológicos sean más complejos o sofisticados (la complejidad de las ideologías se relaciona directamente con la clasificación que aporta Rudé sobre ideologías derivadas y que hemos expuesto más arriba). La complejidad o sofisticación de una ideología se expresa en las prácticas sociales, a través de los mecanismos propuestos para la interacción de los individuos y la densidad de los contenidos que se intercambian. Estos contenidos suelen ser más complejos en las sociedades desarrolladas, cuyo bienestar depende de requerimientos individuales de mayor dificultad para su acceso u obtención.

Una tercera y última consecuencia respecto al planteamiento hecho por van Dijk y su concepción de las ideologías, nos parece muy interesante de abordar en profundidad en un proyecto de investigación amplio y sistemático. Esta consecuencia se refiere a que las ideologías se presentan en manifestaciones contextuales variables. En la actualidad, la variación contextual se manifiesta frecuentemente en individuos que pertenecen a diversos grupos y en tal sentido, su conocimiento y experiencia respecto de sistemas de creencias puede ser también muy diferente. En la práctica, compartir distintas ideologías y valores que pueden ser contradictorios, se traduce en una eventual dificultad para la conformación de redes de coopera- 
ción mutua, obstaculizando con ello el desarrollo de capital social frente a los desafíos de una comunidad o sociedad.

De los planteamientos expuestos aquí que caracterizan una estructura ideológica en la perspectiva de van Dijk (2008), utilizados para enriquecer la comprensión del desarrollo de capital social, podemos concluir que toda ideología es un marco de cognición social compartido por un grupo. Dichos marcos se componen a su vez por valores socioculturales mediante los cuales el grupo se define a sí mismo y establece ciertas fronteras con otros grupos. Para nuestro artículo, lo más relevante de este aporte, es que las ideologías tendrían la función cognitiva de organizar todos los contenidos que le son propios y de esta forma, pueden seguir de manera indirecta, las prácticas sociales de un grupo. Esto último se expresa en la constitución de las redes de intercambio y el tipo de reciprocidad que se establece entre ellas. Su aplicación reiterada de manera exitosa es la que en última instancia va generando capital social, y como hemos venido diciendo a lo largo de este trabajo, el resultado de esa interacción exitosa se verá expresado en el valor de la confianza. Desde nuestra perspectiva, este valor final, el de la confianza, es el que asegura el incremento de capital social, pero no debemos olvidar que su expresión y existencia se originan en una estructura ideológica bien definida, cuyos contenidos, aún cuando evolucionan y sufren modificaciones, representan el referente conceptual básico para un exitoso intercambio social en términos de desarrollo cooperativo.

\section{Reflexiones finales a modo de conclusión}

Como hemos podido observar en este artículo, el concepto de capital social ha tomado gran importancia en el ámbito de las teorías del desarrollo, durante las últimas tres décadas. Su descripción y explicación proviene de dominios disciplinares tales como la sociología y la economía, aportando cada una de ellas, múltiples variables que interactúan fundamentalmente en dos espacios. El primero tiene que ver con un sistema complejo de contenidos que expresan los sistemas de creencias o ideologías, desde las cuales se genera el capital social, y el segundo se relaciona con la constitución efectiva de las redes de cooperación y reciprocidad por medio de las cuales el capital social se incrementa y se hace efectivo.En este trabajo de generación de conocimiento en el ámbito del desarrollo social, concordamos con Portes (1998) en que no es posible aún afirmar que estamos frente a una nueva teoría, y más bien se puede decir que se intenta articular un paradigma emergente cuyas bases epistémicas se encuentran en los trabajos fundacionales de la sociología y de la antropología (como hemos señalado antes a propósito de los aportes de Mauss sobre el concepto de reciprocidad).Precisamente, en este diseño y trazado teórico incompleto, nos ha interesado detenernos en una variable que nos parece básica y esencial para la constitución de capital social. Más allá de las diferencias disciplinares o de perspectivas, desde las cuales se describa el capital social, todos los autores concuerdan en la necesidad de la existencia de un conjunto de valores, normas y reglas desde las cuales establecer las redes 
sociales para el desarrollo comunitario o en niveles más amplios y complejos de organización humana. Desde nuestra perspectiva, dicho consenso exige una revisión de los sistemas de creencias e ideologías que regulan y monitorean el funcionamiento de las redes de relaciones para el desarrollo. Esta consideración cobra mayor relevancia en un contexto mundial en donde la globalización ha sido sinónimo de una acción de territorialización del proyecto neoliberal, territorialización devastadora de sistemas de creencias locales que posibilitan el desarrollo de un capital social vinculado a los elementos más significativos de una cultura en particular.

Estas reflexiones finales, pretenden enfatizar la importancia de los ejes ideológicos a partir de los cuales se puede constituir capital social, precisamente porque nos preocupa la instalación descontrolada del aparato neoliberal en la sociedad planetaria. En este sentido, se ha creado una relación tensa entre este neomercantilismo que regula la vida del hombre moderno, y ciertas reservas de capital social en donde se incluyen sistemas de creencias que rescatan contenidos culturales que circulan desde tiempos remotos, adaptándose a las nuevas condiciones de vida. En este contexto, los sistemas de significación desde los cuales se construye el capital social protegen un nomos esencial que resguarda el sentido antropológico de la existencia del hombre en espacios naturales y culturales en que ha vivido y construido con el tiempo. En los términos en que hemos desarrollado este trabajo, podríamos agregar que la existencia de sistemas ideológicos con capacidad de adaptación y al mismo tiempo vigorosos y densos respecto a sus contenidos fundamentales, representan verdaderas fuerzas de resistencia en relación a un nuevo orden social articulado desde las bases del neoliberalismo económico. La mercadización promovida por las tendencias políticas de la derecha actual sólo se orienta a un consumismo hiperactivo que supera cualquier racionalidad para la satisfacción de las necesidades reales del hombre y su vida en sociedad. En tal dirección, si la preocupación de la sociología del desarrollo se ha orientado a comprender las formas de aseguramiento del ejercicio democrático de las diferentes estructuras y niveles societales, a través de la constitución e incremento de capital social entendido como un activo esencial para la evolución armónica del hombre en contextos comunitarios, las políticas neoliberales amenazan seriamente a la democracia como ejercicio político amplio, y a las democracias reales que funcionan en contextos también reales haciendo esfuerzos para una mayor autogestión de los individuos organizados comunitariamente. El principio de solidaridad, tan propio de la creación de capital social entre los más pobres, resulta quebrantado por un neoliberalismo a ultranza que inocula en el individuo un sentido de competencia exacerbadamente individualista cuya finalidad última tiene que ver con el hiperconsumo al que ya nos referíamos. Dicha dinámica, empobrece la mirada horizontal en una red de relaciones cooperativas y simultáneamente implica la pérdida de las competencias del sujeto, para interactuar con otros, reconocerse a sí mismo y las diferencias que en esa interacción se hacen visibles, aprovechándolas para iniciar un diálogo intergrupal de carácter cooperativo. 
De este modo, desde nuestra perspectiva la globalización económica atenta en contra de la articulación de redes solidarias y del ejercicio democrático por parte de la sociedad civil, provocando crisis financieras que se expanden en niveles micro y macroeconómicos. El sistema ideológico dominante, llamado democracia del mercado, se globaliza de este modo, sin considerar y menos respetar las dinámicas sociales generadas desde espacios culturales específicos en los que se han producido también reglas del juego ad hoc para su funcionamiento. Lo anterior daña severamente el funcionamiento de las bases democráticas interferidas por una sociedad global cuyo proyecto subordina la condición humana a las relaciones de intercambio puramente comercial. Desde la perspectiva neoliberal, la articulación de relaciones sociales cooperativas debe ser entendida como una modalidad más de mercancía afecta a un valor de propiedad e intercambio económico en el que las condiciones de existencia (como el respeto de los derechos humanos) no resultan significativas.

Lo que se intenta instaurar en nuestras sociedades, es una ideología de mercado que amenaza con destruir los fundamentos esenciales de la democracia, es decir, de un sistema de creencias, de una ideología básicamente participativa para la toma de decisiones respecto a los proyectos de desarrollo social. En consecuencia, el libre mercado instala una forma de funcionamiento de los pueblos, que se restringe a la dimensión comercial. Esta forma de funcionamiento y por tanto de ordenamiento de las sociedades ignora las múltiples variables culturales y naturales que van interactuando en la construcción de un nomos cultural rico y complejo que se expresa en un sentido denso de la vida humana organizada en comunidad. Al respecto, la identidad colectiva, el sentido de pertenencia y los vínculos de solidaridad que están asociados al capital social, son valores que no se encuentran en el repertorio ideológico del neoliberalismo.

Las reflexiones antes expuestas, nos vuelven al tema central de este artículo, es decir, a la importancia de un sistema ideológico definido, desde el cual se constituye y desarrolla el capital social. Como hemos señalado en otro espacio de reflexión (Brower 2010), nos interesa la existencia de sistemas de significación ideológica como el conjunto de contenidos que organiza la vida del hombre en sociedad. Estos sistemas se expresan como gramáticas fundamentales a partir de las cuales se produce un gran número de prácticas sociales, entre las que destacan la articulación de redes cooperativas para el desarrollo. Nuestro interés tiene que ver con que esos sistemas de significación en los que se encuentran valores, creencias y normas de organización, sigan existiendo desde los espacios particulares en que han sido concebidos, con las naturales adaptaciones de otras visiones de mundo que puedan enriquecer y potenciar proyectos ideológicos específicos. Del mismo modo hemos hecho referencia, en estas reflexiones finales, al neoliberalismo, advirtiendo sobre su instalación planetaria en el contexto de la globalización. Esta advertencia nos parece altamente significativa pues la sociedad de mercado introduce niveles de incertidumbre que atentan contra el valor de la confianza y la solidaridad, valores centrales para el incremento de capital social. La generación de capital social en este con- 
texto neoliberal globalizado atenta, en efecto, contra la constitución de redes de cooperación entre los más desposeídos que se organizan en comunidades locales muchas veces para reducir los costos de transacciones comerciales. Del mismo modo, la instalación de las sociedades de mercado apunta a la producción en el ámbito privado reduciendo la existencia de bienes públicos de fácil acceso y disposición. En este sentido, el tipo de vinculaciones que se desprenden de las reglas del juego mercantil se establecen más bien, entre los individuos y los grandes conglomerados económicos transnacionales, subordinando la articulación de organizaciones para la gestión de base, cuyos actores principales son los miembros de las sociedades civiles. Es en este espacio, en donde se establece el ejercicio democrático validado por las propias comunidades y por ende es en dicho espacio en donde se expresa el sistema de creencias y valores que dan cuerpo a las ideologías, ejes fundamentales para validar el activo constituido por el capital social.

Como consecuencia de lo anterior, nos resulta de gran relevancia que los sujetos que componen la sociedad civil, en cualquiera de sus niveles, fortalezcan sus competencias políticas, cuyo base y patrimonio es precisamente el capital social y el consecuente éxito de las acciones colectivas en torno a un proyecto compartido de desarrollo para el futuro.

Las teorías sociológicas desarrollistas no deben perder de vista la necesidad de contar con una aproximación formal y sistemática a los dispositivos ideológicos desde los cuales se establecen las redes cooperativas. Ellas no sólo tienen un carácter instrumental orientado a la consecución de objetivos materiales, sino que además representan sistemas de significación simbólica complejos, tejidos durante largos períodos y con la participación de múltiples actores en escenarios sociales que también se han ido modificando, en torno a contenidos valóricos que en su conjunto diseñan un sentido de pertenencia y por ende de identidad. Finalmente y en consonancia con lo ya expresado, el capital social, es mucho más que un conjunto de relaciones articuladas por diferentes formas de protocolos culturales y su valor depende en buena medida de la existencia de una visión de mundo compartida por un grupo de individuos organizados comunitariamente. Esa visión de mundo no sólo es importante para los miembros del grupo y su reconocimiento interno, sino que además, es fundamental para la interacción con otros grupos, en espacios interculturales en donde el diálogo es entre sistemas de creencias distintos. Allí cobran aún mayor relevancia las ideologías particulares, pues cualquier diálogo será más fructífero en la medida en que esos sistemas de creencias establezcan valores y normas definidas y compartidas para un trabajo dialógico en el ámbito de la cooperación. 


\section{Notas}

${ }^{1}$ No podemos dejar de mencionar la gran influencia antropológica de la definición actual de capital social. La reciprocidad, como elemento esencial de este tipo de relaciones, se encuentra ya descrita por Mauss en su célebre Ensayo sobre el Don. Escrito a comienzos del siglo XX, este texto caracteriza la reciprocidad como un principio básico de las relaciones institucionales tanto formales como informales en el ámbito de una comunidad. Del mismo modo establece que ya en las sociedades premercantiles operaba un sistema de intercambio que incluía desde obsequios hasta elementos de ayuda para la subsistencia. Cf. M. Mauss (1990), The Gift: the Form and Reason for Exchange in Archaic Societes.

${ }^{2}$ Respecto al conocimiento del otro, y por tanto, al reconocimiento del espacio propio y desde allí, a las posibilidades de establecer redes de reciprocidad, Bourdieu señala "..., parce que 'connus', d'être connus (cf. "je l'ai bien cornu”), sont en mensure de transformer toutes les relations circonstancielles en liaisons durables". (Bourdieu 1980: 2) "Le capital social”. En Actes de la recherche en sciences sociales.

${ }^{3}$ En relación a estos contenidos morales y éticos, Fukuyama señala que la fuente de la confianza tiene que ver con obligaciones morales recíprocas. Para este autor, tanto la democracia como el capitalismo pueden funcionar de manera correcta, sólo si coexisten con estos contenidos que pueden ser premodernos, pero que aseguran que las reglas del juego establecidas en los contratos y la racionalidad económica se cumplan en beneficio de los grupos sociales implicados. Cf. F. Fukuyama. (1996) Confianza (Trust).

${ }^{4}$ Las otras dos formas de expectativas son la adquisición de competencias y habilidades para cumplir con las demandas establecidas en las relaciones de intercambio, y por otro lado, el nivel de cumplimiento de las obligaciones y responsabilidades contraídas por las partes implicadas. Cf. B. Barber. (1983). The Logic and Limits of Trust.

${ }^{5}$ Nuestro planteamiento de programas de acción incluidos en la estructura ideológica, son concebidos por Friedmann como un repertorio de acciones que necesariamente debe ser conocido por quienes interactúan en diferentes niveles de la sociedad. Este repertorio se vincula directamente con el ideario básico enriquecido por la experiencia, y de ninguna manera debiera corresponder a una retórica improvisada, que puede llevar al fracaso de las interacciones sociales que buscan diferentes formas de bienestar social. Cf. J. Friedmann. (1996). Empowerment: The Politics of Alternative Development. 


\section{Bibliografía}

Aebischer, V., Deconchy, J.P. y Lipiansky, E.M. (1991), Idéologies et représentations sociales. Delval, Fribourg.

Adler, P. y Kwon, S-W. (1999), “Social Capital: The Good, The Bad, and The Ugly”. www.worldbank.org/poverty/scapital/library/papers.htm. Acceso 20/3/2009.

Barber, B. (1983), The Logic and Limits of Trust. New Jersey, Rutgers University Press.

Bourdieu, P. (1980), “Le capital social”. In Actes de la recherche en sciencies sociales. Vol. 31, janvier. pp. 2-3.

Ídem (1985), “The Forms of Capital”. In Handbook of Theory and Research for the Sociology of Education. Ed. J. Richardson, Greenwood, N.Y.

Button, G. (1991), Ethnomethodology and the Human Sciences. Cambridge University Press, Cambridge.

Brower, J. (2010), "Reflexiones Teóricas Sobre la Dimensión SemióticoDiscursiva de la Ideología Política”. Revista RE-Presentaciones. Escuela de Periodismo. Universidad de Santiago. Año 3, Nº6. pp. 61-72.

Coleman, J.S. (1990), Foundations of social theory. Harvard University Press, Cambridge.

Fukuyama, F. (1996), Confianza (Trust). Editorial Atlántida, Buenos Aires.

Friedmann, J. (1996), Empowerment: The Politics of Alternative Development. Massachussetts, Cambridge.

Granovetter, M. (1985), "Economic Action and Social Structure: the Problem of Embeddness”. In American Journal of Sociology. Vol. 91, N³. pp. 481-510.

Mauss, M. (1990), The Gift: the Form and Reason for Exchange in Archaic Societes, Norton, N.Y..

North, D. (1990), Institutions, Institutional Change and Economic Performance. Cambridge University Press, Cambridge, MA, USA.

Portes, A. (1998), “Social Capital: its Origins and Applications in Modern Sociology”. In Annual Review of Sociology, 24:1, pp. 1-24.

Putnam, R. (1993), Para hacer que la democracia funcione, editorial Galac, Caracas. 
Polis, Revista de la Universidad Bolivariana, Volumen 10, $N^{\circ}$ 29, 2011

Rudé, G. (1980), Ideology and Popular Protest. Praeger Publications, New York.

Scott, J. (1985), Weapons of the Weak: Everyday Forms of Peasant Resistance. Yale University Press, New Haven.

Uphoff, N. y Wijayaratna, C.M. (2000), “Demonstrated Benefits from Social Capital: The Productivity of Farmer Organizations in Gal Oya, Sri Lanka”. In World Development. Vol. 28.N¹1. pp. 1875-1890.

Van Dijk, T. (2008), “Semántica del discurso e Ideología”. Revista Discurso y Sociedad, Vol.2 (1). pp. 201-261. www.dissoc.org. Acceso 15/12/2010.

Recibido: 02.05.2011

Aceptado: 06.07.2011 